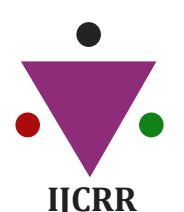

IJCRR

Section: Healthcare

Sci. Journal Impact

Factor: 6.1 (2018)

ICV: 90.90 (2018)

(c) (i) (8)

Copyright@IJCRR

\section{Urinary Thromboxane Production in Diabetic and Non-Diabetic Patients of Acute Coronary Syndrome}

\author{
Sheetal Chauhan ${ }^{1}$, Yeshwanth Rao Karkala ${ }^{2}$, Ajit Singh ${ }^{3}$, Tom Devasia ${ }^{4}$, \\ Hashir Kareem ${ }^{5}$, Deepak Uppunda ${ }^{6}$, Gunajn Bonde ${ }^{7}$
}

'Research Scholar, Department of Pharmacology, Melaka Manipal Medical College, Manipal Academy of Higher Education,

Manipal-576104 India; 'Professor of Pharmacology and Ethics, Saint James School of Medicine, Anguilla (BWI); ${ }^{3}$ Research Associate, Department of Cardiology, Kasturba Medical College, Manipal Academy of Higher Education, Manipal-576104 lndia; ${ }^{4}$ Professor and HOD, Department of Cardiology, Kasturba Medical College, Manipal Academy of Higher Education, Manipal-576104 India; ${ }^{5}$ Associate Professor and Interventional Cardiologist, Department of Cardiology, Kerala lnstitute of Medical Sciences, Trivandrum, Kerala695029, India; ${ }^{6}$ Research Scholar, Department of Cardiology, Kasturba Medical College, Manipal Academy of Higher Education, Manipal-576104 India; ${ }^{7}$ Assistant Professor, School of Health Sciences, University of Petroleum and Energy Studies, Dehradun, India.

\title{
ABSTRACT
}

Background: Diabetes mellitus is an independent predictor of higher major adverse cardiac events (MACE) rate following percutaneous transluminal coronary angioplasty (PTCA) in acute coronary syndrome (ACS). Higher thromboxane production in diabetic patients has not been linked to aspirin resistance and recurrent events.

Methods: This prospective observational cohort study aimed to investigate urinary 11-dehydro-thromboxane B2 (dh-TxB2) levels in diabetic and non-diabetic ACS patients undergoing PTCA and its association with MACE. The dh-TxB2 ELISA assay was performed during index admission in all patients, and they were followed-up for 1-year.

Results: Out of the 192 patients enrolled, 84 (43.8\%) were diabetic and 108 (56.2\%) were non-diabetic. Median dh-TxB2 levels were higher among diabetics compared to non-diabetics [577.7 pg/mg Cr (IQR 991.2:62.8-1054.0) vs. 344.0 pg/mg Cr (IQR 691.4:73.4-764.8) respectively]. Patients with and without diabetes had 1-year MACE rates of $14.3 \%$ and $7.4 \%$ respectively $(\mathrm{P}=0.096)$. The patients who developed MACE had higher dh-TxB2 levels in both the groups [nondiabetics: 419.6 (non-MACE) vs. $1349.7 \mathrm{pg} / \mathrm{mg} \mathrm{Cr}$ (MACE); $p<0.001$ ] [diabetics: 647.7 (non-MACE) vs. $1346.5 \mathrm{pg} / \mathrm{mg} \mathrm{Cr}$ (MACE); $p=0.029$ ].

Conclusion: Patients with DM had higher dh-TxB2 levels within 24 hours of PTCA despite similar aspirin therapy. Diabetic ACS patients who have the concentration of dh-TxB2 more than $>1496 \mathrm{pg} / \mathrm{mg} \mathrm{Cr}$ developed more MACE than their counterparts in non-diabetics. The dh-TxB2 concentration may be used as a predictor of early MACE in diabetic ACS patients.

Key Words: Acute coronary Syndrome, Diabetes Mellitus, Dehydro-thromboxane B2, Major Adverse Cardiac Events, Percutaneous Transluminal Coronary Angioplasty

\section{INTRODUCTION}

Acute coronary syndrome (ACS) is a state of ischemic cardiac abnormalities and results from accelerated atherosclerosis process ${ }^{1}$. The simultaneous presence of the risk factor contributes to enhanced morbidity and mortality in these patients. Diabetes is one of the most frequent comorbidities in patients with heart disease and leads to increased atherothrombotic risk ${ }^{2,3,4}$. Landmark trials showed that around 15$40 \%$ of patients with ACS have a background of diabetes ${ }^{5}$.
Further, diabetes is associated with a 2- to 4-fold higher risk of cardiovascular disease (CVD), and diabetic patients have two-timed more risk of mortality compared to non-diabetic patients $^{5,6}$. The presence of diabetes is a strong independent predictor of short-term and long-term recurrent ischemic events in ACS patients after percutaneous transluminal coronary angioplasty (PTCA) $)^{3,4}$. The Hyperglycemic state caused by DM increases platelet aggregation, adhesion, and platelet turnover along with increased production of p-selectin, prostacyclin, nitric oxide, and thromboxane. These factors

\section{Corresponding Author:}

Dr. Yeshwanth Rao Karkala, MD, Professor of Pharmacology and Ethics, Saint James School of Medicine, Anguilla (BWI). Email: yashwanthrao2000@gmail.com; yrao@mail.sjsm.org; ORCID ID: 0000-0002-1448-2851

ISSN: 2231-2196 (Print)

Received: 17.09 .2020
ISSN: $0975-5241$ (Online)

Revised: 03.10 .2020
Accepted: 11.10 .2020
Published: 27.10 .2020 
play a crucial role in the atherothrombotic process in highrisk patients ${ }^{5,7,8}$. Thromboxane is a potent platelet aggregation factor and excreted in the urine in the form of 11-dehydro-thromboxane B2 (dh-TxB2). It is a stable metabolite of thromboxane A2, which indicates thromboxane production and platelet activation in $v i v o^{9,10}$. Previous studies showed that diabetic patients produce more thromboxane as compare to healthy subjects ${ }^{5,8}$. In a very few studies, diabetic patients who were on aspirin treatment showed high thromboxane levels as an independent risk factor for future major adverse cardiac events (MACE) $)^{11,12}$.

The majority of the studies investigated aspirin dose-response to inhibit platelet hyperactivity using platelet function assays in coronary artery disease (CAD) patients. The light transmission aggregometry was widely used in the past decades but is unable to estimate dh-TxB2 directly ${ }^{2,11,14}$. Urinary dhTxB2 measurement is a direct and more sensitive method to evaluate in-vivo platelet activation. The studies on direct estimation of dh-TxB2 are limited in the high-risk ACS population ${ }^{2,11}$. Therefore, the present study aimed to assess the effect of diabetes on thromboxane production in patients with ACS who underwent percutaneous transluminal coronary angioplasty with drug-eluting stents (DES) and its association with one-year outcomes.

\section{METHODS}

\section{Information Disclosure}

This prospective cohort study has included 192 patients with ACS who underwent PTCA at our tertiary care center from May 2017 to April 2018. The study protocol was cleared from the institutional ethics committee and further written informed consent was taken to all recruited patients for participating and sample collection under the present study. Before starting the study, a brief report was provided for clinical trial registration, and the approval statement was issued in January 2018 (CTRI/2018/01/011578).

\section{Study Design, Population and Baseline Charac- teristics}

All recruited patients were divided into two groups based on the presence of diabetes mellitus (DM). The patients were eligible for inclusion if aged $\geq 18$ years, diagnosed with ACS, prescribed on dual antiplatelet therapy (aspirin along with P2Y12 inhibitor), and successfully underwent PTCA for the first time.

The patients were excluded from the study if: on oral or parenteral anticoagulation, use of non-steroid anti-inflammatory drugs in the last one month, psychiatric illness on medications which can impact patient's behaviour to medication compliance, pregnancy, severe valvular heart disease, histo- ry of active malignancy or chronic inflammatory conditions, cancer, hemorrhagic diathesis, liver cirrhosis, severe renal disease, patient's refusal to participate in the study. Other exclusion criteria include haemoglobin levels $<10 \mathrm{~g} / \mathrm{dL}$ (both males and females), platelets $<100,000 / \mathrm{mm}^{3}$ or $>500,000 /$ $\mathrm{mm}^{3}$, serum creatinine $>1.2 \mathrm{mg} / \mathrm{dl}$, and patients with ejection fraction $<40 \%$.

All the patients were loaded with dual antiplatelet therapy (DAPT), aspirin $325 \mathrm{mg}$ with ticagrelor $180 \mathrm{mg}$, or clopidogrel $300 \mathrm{mg}$ at the patient's arrival to the hospital. At hospital discharge, they were prescribed to take aspirin (75 $\mathrm{mg}-150 \mathrm{mg}$ ) with ticagrelor $180 \mathrm{mg}$ or clopidogrel $(75 \mathrm{mg}-$ $150 \mathrm{mg}$ ) daily and instructed to take medicines on scheduled time until hospital re-visit or unless directed otherwise by a physician.

At the time of enrolment in the study, demographics characteristics, data on comorbidities, history of presenting complaints, medical history, and medication history were collected for the subjects using standardized data collection forms. Every crucial aspect of coronary interventions and cardiovascular symptoms were analyzed.

\section{Sample Collection and dh-TxB2 estimation}

For diabetic and non-diabetic patients who participated in the study, blood samples were collected by clean venipuncture to perform standard laboratory tests at index hospitalization. Further, for every patient, urine samples were also collected for dh-TxB2 measurement. The first-morning urine sample was collected within 24 hours of post-angioplasty and immediately stored at $-80^{\circ} \mathrm{C}$ for future analysis. The supernatant was used for further processing after centrifuging the sample at $1000 \mathrm{~g}$ for 15 minutes. The concentrations of metabolite dh-TxB2 were measured using a commercially available ELISA assay (Assay \#519510, Cayman Chemical, Ann Arbor, MI, USA), according to the manufacturer's instructions. The assay was performed within three months of sample collection with the help of the colourimetric method. The dh-TxB2 concentrations were normalized to urinary creatinine concentrations, and results were expressed as a ratio, in picograms of dh-TxB2 per milligram of urine creatinine (pg/mg Cr). The cut-off value of dh-TxB2 was calculated $\geq 1495.8 \mathrm{pg} / \mathrm{mg} \mathrm{Cr}$ as per the receiver operating characteristic (ROC) curve.

\section{Follow-up and Study Outcome Measurements}

All the patients were followed up for 12-months after recruitment for primary and secondary clinical outcomes. Primary outcomes were compared between both groups in the form of composite MACE. For the current study, MACE was defined as a composite of myocardial infarction [unstable angina, non-ST segment elevation myocardial infarction (NSTEMI), ST-segment elevation myocardial infarction 
(STEMI)], stroke, target vessel revascularization (TVR), target lesion revascularization (TLR), and cardiovascular and/ or all causes death. Heart failure (HF) was presented as a secondary outcome, and patients who developed HF were compared separately from MACE.

\section{Statistical Analysis}

Categorical variables were expressed as proportions, and continuous variables were reported as mean $\pm \mathrm{SD}$ or median, depending on their distribution. Proportions were compared using the chi-square test and Fisher exact test. Continuous variables were compared by Student $t$-test or paired t-tests for normally distributed data and Independent samples median test. The impact of DM and explanatory variables were analyzed using Cox's proportional hazards regression model. Univariate logistic regression analysis was performed to evaluate the associations between DM and dh-TxB2 and MACEs. Further optimized multivariable Cox proportional hazard models were used to identify the independent predictors that correlated with diabetic ACS patients. Time-topoint survival was presented using the Kaplan-Meier curve. The statistical analyses were performed with SPSS software version 22.0 and $\mathrm{P}$ - values $<0.05$ were considered to be statistically significant.

\section{RESULTS}

Out of the total, $108(56.2 \%)$ patients were in the non-diabetic group and $84(43.8 \%)$ in the diabetic group. The majority of patients who have DM were male and had a history of hypertension $(p=0.001)$. Diabetic patients had higher LDL levels than non-diabetic patients. The majority of diabetic patients had the multivessel disease as compared to nondiabetic patients. The patient's baseline clinical and demographics characteristics, disease-related details along with intervention, and medication description were analyzed between defined groups (Table 1).

During the 12-months follow-up period, we observed 10.4\% incidences of composite MACE in recruited patients, $14.3 \%$ in diabetic, and $7.4 \%$ in the non-diabetic group with no significant difference. There were $3.6 \%$ of patients who had $\mathrm{CV}$ death in the diabetic group, while no $\mathrm{CV}$ death was observed in the non-diabetic group (Table 2). Diabetic patients displayed significantly higher concentration of dh-TxB2 in comparison to non-diabetic patients $[577.7 \mathrm{pg} / \mathrm{mg} \mathrm{Cr}\{\mathrm{IQR}$ 991.2 (62.8-1054.0)\} vs. 344.0 pg/mg Cr \{IQR 691.4 (73.4764.8)\} respectively]. Concentration differences of dh-TxB2 were significant between the patients who developed MACE and who did not, in non-diabetic [419.6 (non-MACE) vs. $1349.7 \mathrm{pg} / \mathrm{mg} \mathrm{Cr}$ (MACE); $<0.001]$ and diabetic groups [647.7 (non-MACE) vs. $1346.5 \mathrm{pg} / \mathrm{mg} \mathrm{Cr}$ (MACE); $\mathrm{p}=$ 0.029] (Fig. 1). Patients in diabetic and non-diabetic groups who have a high concentration of dh-TxB2 (>1496 pg/mg $\mathrm{Cr})$ developed more MACE $(33.3 \%)$ and $(50 \%)$, respectively, in comparison to the patients with low 11-dh-TxB2 concentration than the cut-off ( $p<0.05)$.

The independent predictors of composite MACE in diabetic against non-diabetic patients were analyzed (Table 3). Timeto-event curve did not have significant difference between survivals in both the groups [306 (diabetic) vs. 330 days (non-diabetic); $p=0.129$ ] (Fig. 2).

\section{DISCUSSION}

We evaluated the urinary thromboxane production in diabetic ACS patients who underwent PTCA, to understand the impact of thromboxane and diabetes combine immediately after angioplasty. Our study demonstrated that immediately after PTCA, thromboxane production increased in diabetic patients when compared with non-diabetic patients. We also observed that ACS patients who have a high concentration of dh-TxB2 more than cut off value developed more MACE compare to patients with low dh-TxB2 concentration.

In ACS patients, thromboxane production directly reflects platelet activation, and the metabolite is approximately two times higher in comparison to healthy individuals. In patients who underwent PTCA, the 11-dh-TxB2 level increases up to 15-fold within the 2.5 to 5.0 -hours ${ }^{5,15}$. Immediate after PTCA platelets get over-activated and released in high amounts due to intense physiologic stress and inflammation ${ }^{16,17}$. PTCA acts as an acute and local stimulus to activate platelets, which is an important factor for recurrent events. Further, clinical factors also affect platelet activity, including diabetes ${ }^{11}$.

Earlier studies showed that DM is associated with short and long-term adverse clinical outcomes ${ }^{4}$. The recurrence rate of ischemic events is high in diabetic patients compared to healthy individuals. In these patients, platelets have an increased tendency to activate and aggregate, which leads to platelet hyperactivity. Platelet reveals reduced membrane fluidity, which may reflect glycation of membrane proteins and increased metabolism of arachidonic acid in diabetic patients in response to this TxA2 production enhances, which in turn contributes to increased platelet sensitivity ${ }^{2}$, ${ }^{18-20}$. Francesco Zaccardi et al. observed platelet activation in adult subjects with well-controlled Type-1 DM via thromboxane biosynthesis and oxidative stress. They conclude that patients with type-1 DM have persistently enhanced in vivo platelet activation, especially in females ${ }^{21}$. The ASPECT study team investigated platelet functions in diabetic and non-diabetic patients and found that diabetic patients show a high platelet reactivity. The level of dh-TxB2 was significantly higher in diabetic patients $(p=0.02)^{22}$. The current study shows that the concentration of dh-TxB2 was significantly high in diabetic patients against non-diabetic ACS pa- 
tients. The patients who developed MACE in both diabetic and non-diabetic groups have had a significant difference in dh-TxB2 concentrations, too, in comparison to the patients who did not develop MACE. The difference in dh-TxB2 production in MACE vs. non-MACE patients was higher in the non-diabetic group $(p<0.001)$ than the diabetic group $(p=$ 0.029). That suggests that the patients who did not develop MACE in the diabetic group have had higher concentration than in the non-diabetic group. Diabetic patients tend to have increased platelet reactivity whether they develop MACE or not, but the patients are always at high risk of developing early MACE.

In our study, we observed that diabetic ACS patients were associated with worsening clinical outcomes following PTCA and presence of DM increase the risk of mortality [all cause death in diabetic $(5.9 \%)$ vs. non-diabetic $(0.9 \%) ; \mathrm{p}<0.001]$. Luca G. D. et al. also investigated the impact of DM on longterm outcomes in STEMI patients undergoing primary angioplasty either with bare-metal stents (BMS) or DES. This study showed that the DM was associated with high longterm mortality, recurrent MI, and in-stent thrombosis, but there was no difference seen between patients treated with BMS or DES ${ }^{4}$. Yujin Yang et al. also observed the clinical effect of diabetes in CAD patients who underwent contemporary PCI. The study demonstrated that the DM was correlated with poorer clinical outcomes in patients after PCI. $\mathrm{DM}$ was an independent risk factor for adverse clinical outcomes in contemporary stented CAD patients. This study did not show any significant difference in composite MACE between both groups. All the $\mathrm{CV}$ death occurred were in diabetic patients; even all-cause deaths were significantly high in the diabetic population. Heat failure was noticeably insignificant between both the groups ${ }^{23}$. Our study is in agreement with this study, where all-cause death was significantly high in a diabetic group compared to non-diabetic $(\mathrm{p}<0.001)$.

\section{Study limitations}

Our study has few limitations; first, the sample size was small, and it may be a reason for no statistically significant difference in the incidence of MACE between diabetic and non-diabetic groups. However, the recruited cohort was large enough to detect a difference in dh-TxB2 production in diabetic and non-diabetics. Second, we did not perform other platelet function assays and platelet-activating signalling pathways in addition to urinary $\mathrm{TxB} 2$ assay, but it did not hamper the results.

\section{Study strengths}

The Major strength of this study is the very specific cohort, where inclusion and exclusion criteria eliminated potential confounders affecting platelet activation to preclude the interference. We have rejected the ACS patients with a history of low haemoglobin and any blood diathesis, severe liver dysfunction, patients with renal insufficiency. We recruited subjects who have developed ACS for the first time and underwent PCI with DES and started on antiplatelet therapy or any other antithrombotic therapy for the first time. One more strength of the present study includes a very low dropout of patients during a 1-year follow-up as patients were counselled.

\section{CONCLUSION}

Our findings showed that the level of dh-TxB2 among diabetic ACS patients undergoing PTCA was higher in comparison to non-diabetic ACS patients. ACS patients who have a high concentration of dh-TxB2 (more than cut-off value) developed higher MACE. During the 12-months follow-up period, incidences of composite MACE were high in diabetics than in the non-diabetic group, which shows that DM is associated with increased risk of early recurrent ischemic events. These findings suggest some more platelet function testing in DM patients to establish a standard method to evaluate the high risk in ACS patients after angioplasty.

\section{ACKNOWLEDGMENT}

Authors acknowledge the immense help received from the scholars whose articles are cited and included in references to this manuscript. The authors are also grateful to authors / editors / publishers of all those articles, journals, and books from which the literature for this article has been reviewed and discussed.

\section{Conflict of Interest: Nil}

\section{Source of Funding: Nil}

\section{REFERENCES}

1. Smith JN, Negrelli JM, Manek MB, Hawes EM, Viera AJ. Diagnosis and management of acute coronary syndrome: an evidence-based update. The Journal of the American Board of Family Medicine. 2015 Mar 1;28(2):283-93.

2. Angiolillo DJ, Fernandez-Ortiz A, Bernardo E, Ramírez C, Sabaté M, Jimenez-Quevedo P, Hernández R, Moreno R, Escaned J, Alfonso F, Banuelos C. Platelet function profiles in patients with type 2 diabetes and coronary artery disease on combined aspirin and clopidogrel treatment. Diabetes. 2005 Aug 1;54(8):2430-5.

3. Ferreiro JL, Angiolillo DJ. Diabetes and antiplatelet therapy in acute coronary syndrome. Circulation. 2011 Feb 22;123(7):798813.

4. De Luca G, Dirksen MT, Spaulding C, Kelbæk H, Schalij M, Thuesen L, Van Der Hoeven B, Vink MA, Kaiser C, Musto C, Chechi T. Impact of diabetes on long-term outcome after primary angioplasty: insights from the DESERT cooperation. Diabetes Care. 2013 Apr 1;36(4):1020-5.

5. Rossington JA, Brown OI, Hoye A. Systematic review and metaanalysis of optimal P2Y12 blockade in dual antiplatelet therapy 
for patients with diabetes with acute coronary syndrome. Open Heart. 2016 Feb 1;3(1):e000296.

6. Proscia C, Nusca A, Simona M, Rosetta M, Di Sciascio G. Platelet reactivity and antiplatelet management in diabetic patients with coronary artery disease. Interventional Cardiology. 2015 Jul 1;7(3):283.

7. Wisinski JA, Kimple ME. Platelet dysfunction in type 1 diabetes: stressing the thromboxanes. Diabetes. 2016 Feb 1;65(2):349-51.

8. Kakouros N, Rade JJ, Kourliouros A, Resar JR. Platelet function in patients with diabetes mellitus: from a theoretical to a practical perspective. International journal of endocrinology. 2011;2011.

9. Lopez LR, Guyer KE, De La Torre IG, Pitts KR, Matsuura E, Ames PR. Platelet thromboxane (11-dehydro-Thromboxane B2) and aspirin response in patients with diabetes and coronary artery disease. World journal of diabetes. 2014 Apr 15;5(2):115.

10. Neath SX, Jefferies JL, Berger JS, Wu AH, McConnell JP, Boone JL, McCullough PA, Jesse RL, Maisel AS. The current and future landscape of urinary thromboxane testing to evaluate atherothrombotic risk. Reviews in cardiovascular medicine. 2019 Jun 14;15(2):119-30.

11. Zhang JJ, Gao XF, Ge Z, Tian NL, Liu ZZ, Lin S, Ye F, Chen SL. High platelet reactivity affects the clinical outcomes of patients undergoing percutaneous coronary intervention. BMC cardiovascular disorders. $2016 \mathrm{Dec} ; 16(1): 240$.

12. Pastori D, Pignatelli P, Farcomeni A, Cangemi R, Hiatt WR, Bartimoccia S, Nocella C, Vicario T, Bucci T, Carnevale R, Lip GY. Urinary 11-dehydro-thromboxane B2 is associated with cardiovascular events and mortality in patients with atrial fibrillation. American heart journal. 2015 Sep 1;170(3):490-97.

13. Pulcinelli FM, Biasucci LM, Riondino S, Giubilato S, Leo A, Di Renzo L, Trifiro E, Mattiello T, Pitocco D, Liuzzo G, Ghirlanda G. COX-1 sensitivity and thromboxane A2 production in type 1 and type 2 diabetic patients under chronic aspirin treatment. European heart journal. 2009 May 1;30(10):1279-86.

14. Lemkes BA, Bähler L, Kamphuisen PW, Stroobants AK, Van Den Dool EJ, Hoekstra JB, Nieuwland R, Gerdes VE, Holleman F. The influence of aspirin dose and glycemic control on platelet inhibition in patients with type 2 diabetes mellitus. Journal of Thrombosis and Haemostasis. 2012 Apr;10(4):639-46.

15. Simeone P, Boccatonda A, Liani R, Santilli F. Significance of urinary 11-dehydro-thromboxane b2 in age-related diseases: Focus on atherothrombosis. Ageing research reviews. 2018 Dec 1;48:51-78.

16. Tscharre M, Farhan S, Bruno V, Rohla M, Egger F, Weiss TW, Hubl W, Willheim M, Wojta J, Geppert A, Huber K. Impact of platelet turnover on long-term adverse cardiovascular outcomes in patients undergoing percutaneous coronary intervention. European journal of clinical investigation. 2019 Sep;49(9):e13157.

17. Vejar M, Fragasso G, Hackett D, Lipkin DP, Maseri A, Born GV, Ciabattoni G, Patrono C. Dissociation of platelet activation and spontaneous myocardial ischemia in unstable angina. Thrombosis and haemostasis. 1990 Oct;64(02):163-8.

18. Colwell JA, Nair RM, Halushka PV, Rogers C, Whetsell A, Sagel J. Platelet adhesion and aggregation in diabetes mellitus. Metabolism. 1979 Apr 1;28(4):394-400.

19. Halushka PV, Rogers RC, Loadholt CB, Colwell JA. Increased platelet thromboxane synthesis in diabetes mellitus. The Journal of laboratory and clinical medicine. 1981 Jan;97(1):87-96.

20. Colwell JA, Nesto RW. The platelet in diabetes: focus on prevention of ischemic events. Diabetes care. 2003 Jul 1;26(7):2181188.

21. Zaccardi F, Rizzi A, Petrucci G, Ciaffardini F, Tanese L, Pagliaccia F, Cavalca V, Ciminello A, Habib A, Squellerio I, Rizzo P. In vivo platelet activation and aspirin responsiveness in type 1 diabetes. Diabetes. 2016 Feb 1;65(2):503-9.

22. DiChiara J, Bliden KP, Tantry US, Hamed MS, Antonino MJ, Suarez TA, Bailon O, Singla A, Gurbel PA. The effect of aspirin dosing on platelet function in diabetic and non-diabetic patients: an analysis from the aspirin-induced platelet effect (ASPECT) study. Diabetes. 2007 Dec 1;56(12):3014-9.

23. Yang Y, Park GM, Han S, Kim YG, Suh J, Park HW, Won KB, Ann SH, Kim SJ, Kim DW, Park MW. Impact of diabetes mellitus in patients undergoing contemporary percutaneous coronary intervention: Results from a Korean nationwide study. PloS one. 2018;13(12): e0208746.

\section{Table 1: Baseline characteristics}

\begin{tabular}{|c|c|c|c|}
\hline Variables & Non-diabetic $(n=108)$ & Diabetic $(n=84)$ & p-value \\
\hline \multicolumn{4}{|c|}{ Scio-demographic and clinical characteristics } \\
\hline Age & $58.54 \pm 11.66$ & $59.09 \pm 10.07$ & 0.728 \\
\hline \multicolumn{4}{|l|}{ Gender } \\
\hline Male & $76(54.3 \%)$ & $64(45 \cdot 7 \%)$ & 0.231 \\
\hline Female & $32(61.5 \%)$ & $20(38.5 \%)$ & \\
\hline Smoking & $34(31.5 \%)$ & $29(34.5 \%)$ & 0.536 \\
\hline Hypertension & $49(45 \cdot 4 \%)$ & $57(67.9 \%)$ & 0.001 \\
\hline Dyslipidaemia* & $91(56.9 \%)$ & $69(43.1 \%)$ & 0.421 \\
\hline Ejection Fraction (EF) & $56.7 \pm 7.9$ & $56.2 \pm 8.5$ & 0.680 \\
\hline Thyroid Dysfunction & $5(4.6 \%)$ & $6(7.1 \%)$ & 0.331 \\
\hline \multicolumn{4}{|c|}{ Disease type } \\
\hline Unstable angina & $16(14.8 \%)$ & $25(29.8 \%)$ & 0.014 \\
\hline STEMI & $79(73.1 \%)$ & $45(53.6 \%)$ & \\
\hline NSTEMI & $13(12.0 \%)$ & $14(16.7 \%)$ & \\
\hline Single Vessel Disease & $42(38.9 \%)$ & $29(34.5 \%)$ & 0.251 \\
\hline Multi Vessel Disease & $66(61.1 \%)$ & $55(65 \cdot 5 \%)$ & \\
\hline
\end{tabular}


Table 1: (Continued)

\begin{tabular}{|c|c|c|c|}
\hline Variables & Non-diabetic $(n=108)$ & Diabetic $(\mathbf{n}=\mathbf{8 4})$ & p-value \\
\hline \multicolumn{4}{|c|}{ Lab investigations } \\
\hline Platelet Count & $276.67 \pm 74.23$ & $258.28 \pm 77.33$ & 0.096 \\
\hline Platelet Distribution Width & $16.66 \pm 0.62$ & $16.78 \pm 0.68$ & 0.195 \\
\hline Mean Platelet Volume & $8.18 \pm 0.83$ & $8.38 \pm 0.96$ & 0.103 \\
\hline Haemoglobin & $14.16 \pm 3.14$ & $13.88 \pm 2.11$ & 0.480 \\
\hline \multicolumn{4}{|c|}{ Medications during the index hospitalization } \\
\hline Aspirin ${ }^{*}$ & $106(98.1 \%)$ & $84(100.0 \%)$ & 0.315 \\
\hline Ticagrelor & $94(87.0 \%)$ & $79(94.0 \%)$ & 0.114 \\
\hline Clopidogrel & $13(12.0 \%)$ & $5(6.0 \%)$ & 0.117 \\
\hline Statins & $108(100.0 \%)$ & $83(98.8 \%)$ & 0.082 \\
\hline Beta blockers & $39(36.1 \%)$ & $37(44.0 \%)$ & 0.167 \\
\hline ACE-inhibitors/ARBs & $42(38.9 \%)$ & $28(33.3 \%)$ & 0.452 \\
\hline
\end{tabular}

STEMI ST-elevated myocardial Infarction, NSTEMI non-ST-elevation myocardial infarction, RCA right coronary artery, LAD left anterior descending artery, LCx left circumflex coronary artery, ACE angiotensin converting enzyme, ARB angiotensin receptor blockers *Dyslipidemia defined as total cholesterol $>250 \mathrm{mg} / \mathrm{dL}$, Low density lipids (LDL) >130 mg/dL, High density lipids (HDL) <40 mg/dL $(<50 \mathrm{mg} / \mathrm{dL}$ for women) in the fasting state

"Loading dose of antiplatelet drugs was administered to all 192 patients (Aspirin loading dose $=325 \mathrm{mg}$; Clopidogrel loading dose= $300 \mathrm{mg}$ and Ticagrelor $=18 \mathrm{o} \mathrm{mg}$ )

Table 2: Composite outcomes in diabetic and non-diabetic acute coronary syndrome patients

\begin{tabular}{lcccc} 
Variables & Non-diabetic $(\mathbf{n}, \mathbf{1 0 8})$ & Diabetic $(\mathbf{n}, \mathbf{8 4})$ & Total $(\mathbf{n}, \mathbf{1 9 2})$ & p-value \\
Composite MACE & $7(6.5 \%)$ & $13(15.5 \%)$ & $20(10.4 \%)$ & 0.096 \\
All cause death & $1(0.9 \%)$ & $5(5.9 \%)$ & $6(3.1 \%)$ & $<0.001$ \\
Non-fatal MI & $4(3.8 \%)$ & $5(5.9 \%)$ & $9(4.7 \%)$ & 0.172 \\
Revascularisation & $2(1.9 \%)$ & $3(3.6 \%)$ & $5(2.6 \%)$ & 0.067 \\
CV death & 0 & $3(3.6 \%)$ & $3(1.5 \%)$ & - \\
Non-ischemic Heart Failure & $4(3.8 \%)$ & $5(5.9 \%)$ & $9(4.7 \%)$ & 0.368 \\
\hline
\end{tabular}

CV cardiovascular, MACE major adverse cardiac events, MI myocardial infarction

Table 3: Univariate and multivariate analysis for correlates of 1-year major cardiac adverse events in diabetic and nondiabetic patients

\begin{tabular}{|c|c|c|c|c|c|c|c|c|}
\hline \multirow[t]{3}{*}{ Variables } & \multicolumn{4}{|c|}{ Univariate analysis } & \multicolumn{4}{|c|}{ Multivariate analysis } \\
\hline & \multicolumn{2}{|l|}{ Diabetic } & \multicolumn{2}{|c|}{ Nondiabetic } & \multicolumn{2}{|l|}{ Diabetic } & \multicolumn{2}{|l|}{ Nondiabetic } \\
\hline & OR $(95 \% \mathrm{CI})$ & $\begin{array}{c}\mathbf{p}- \\
\text { value }\end{array}$ & OR $(95 \% \mathrm{CI})$ & $\begin{array}{c}\text { p- } \\
\text { value }\end{array}$ & OR $(95 \% \mathrm{CI})$ & $\begin{array}{c}\text { p- } \\
\text { value }\end{array}$ & OR $(95 \% \mathrm{CI})$ & $\begin{array}{c}\mathbf{p}- \\
\text { value }\end{array}$ \\
\hline Hypertension & $2.61(0.57,11.94)$ & 0.197 & $1.19(0.29,4.75)$ & 0.809 & $1.58(0.32,7.75)$ & 0.576 & $4.09(3.10,10.75)$ & 0.730 \\
\hline $\mathrm{MVD}^{*}$ & $4.56(1.44,14.41)$ & 0.010 & $3.91(0.98,15.64)$ & 0.054 & $3.21(0.75,13.74)$ & 0.116 & $10.84(0.74,158.77)$ & 0.081 \\
\hline LAD involvement & $3.23(0.42,25.07)$ & 0.234 & $3.10(0.38,25.19)$ & 0.290 & & & & \\
\hline $\begin{array}{l}\text { LAD+LCx In- } \\
\text { volvement }\end{array}$ & $2.77(0.88,8.73)$ & 0.070 & $1.92(0.48,7.68)$ & 0.356 & $1.99(0.49,8.06)$ & 0.332 & $2.81(0.19,40.86)$ & 0.449 \\
\hline Beta-blockers & $0.24(0.05,0.99)$ & 0.048 & $1.09(0.26,4.57)$ & 0.905 & $0.28(0.06,1.44)$ & 0.128 & $0.82(0.17,3.89)$ & 0.804 \\
\hline dh-TxB2 levels ${ }^{* *}$ & $4.09(3.10,10.75)$ & 0.004 & $27.74(6.87,112.06)$ & $<0.001$ & $5.05(1.41,18.43)$ & 0.014 & $148.75(13.01,1701.01)$ & $<0.001$ \\
\hline
\end{tabular}

CI confidence interval, LAD left anterior descending artery, LCx left circumflex coronary artery, dh-TxB2 urinary 11-dehydrothromboxane B2, MVD multi vessel disease, OR odds ratio

*Single vessel vs. multi vessel disease

${ }^{* *}$ High dh-TxB2 levels ( $\geq 1496 \mathrm{pg} / \mathrm{mL}$ serum creatinine) 

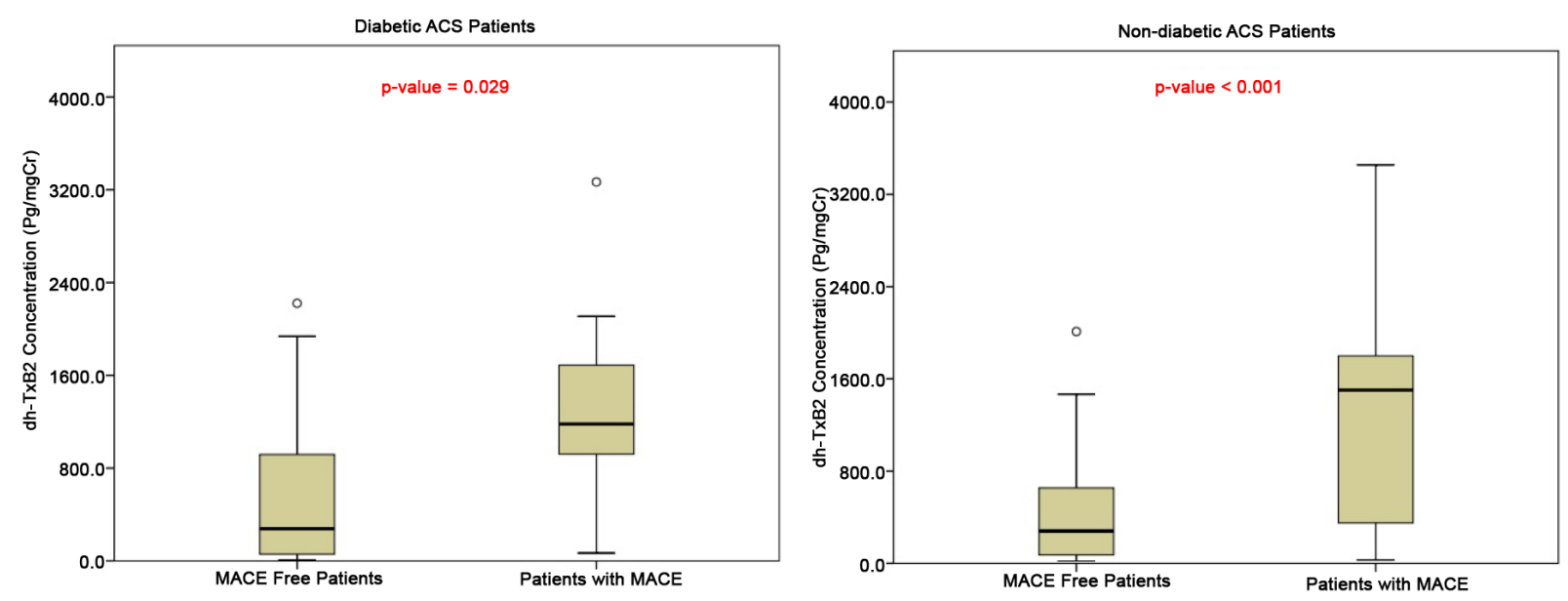

Figure 1: Box-plots showing concentration differences between diabetic (left side) and non-diabetic (right side) ACS patients concerning the development of MACE within 1-year.

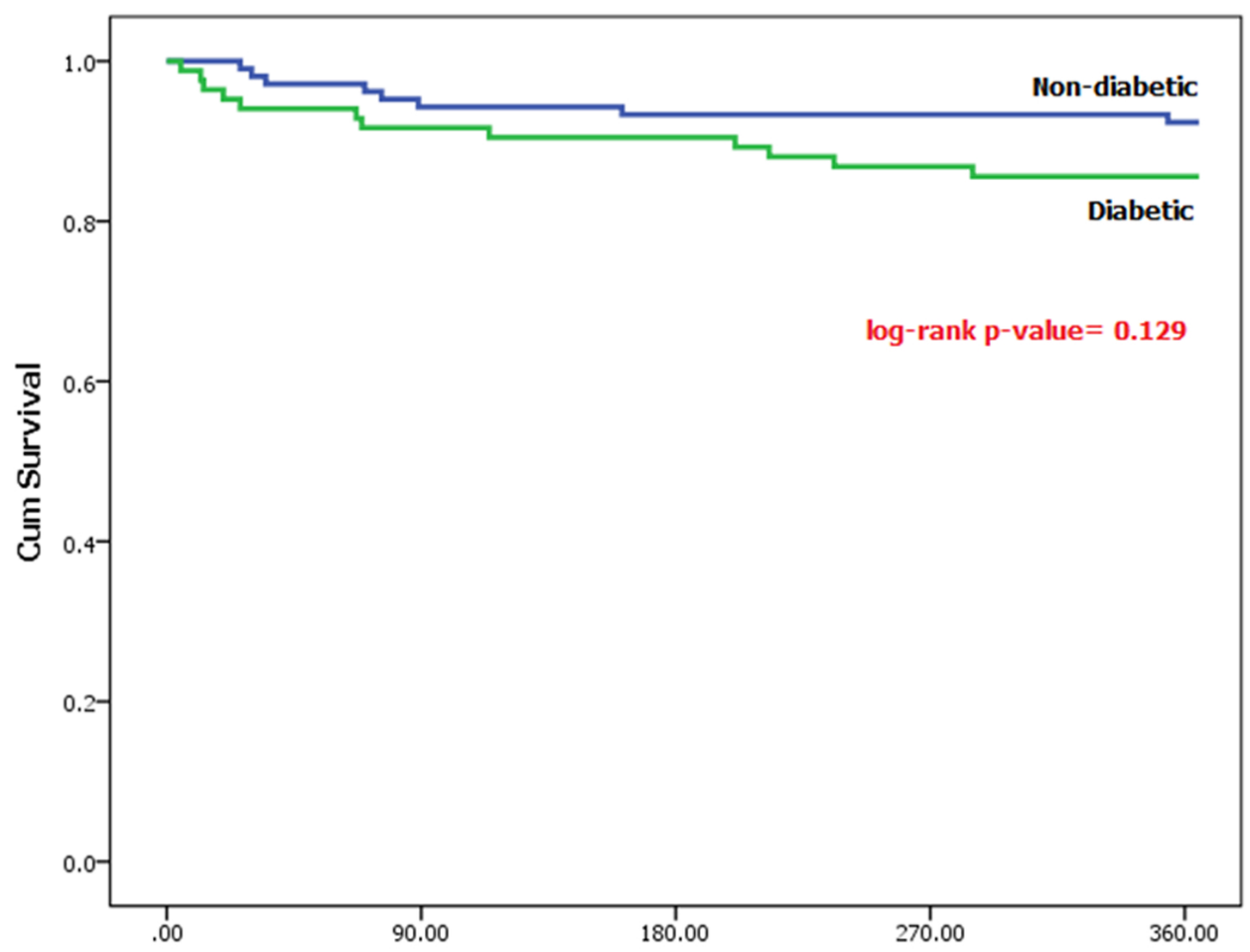

Figure 2: Time-to-event survival analysis at 1-year between diabetic and non-diabetic ACS patients. 